\title{
Attraction Activities of Terrestrial Vegetable Extracts for Aquatic Animals
}

\author{
Katsuhiko Harada, ${ }^{* 1}$ Taiko Miyasaki, ${ }^{* 1}$ and Atsukiyo Karimata*2 \\ *1Department of Food Science and Technology, National Fisheries University, Shimonoseki, \\ Yamaguchi 759-65, Japan \\ ${ }^{*}$ Section of Olericulture Science, Atsugi Central Farm, Tokyo University of Agriculture, \\ Atsugi, Kanagawa 243, Japan
}

(Received October 25, 1995)

\begin{abstract}
Attraction activities of terrestrial vegetables for black abalone Haliotis discus, oriental weatherfish Misgurnus anguillicaudatus, and yellowtail Seriola quinqueradiata were estimated by behavioral experiments in a laboratory test tank as well as an attraction index $a$ for both abalone and oriental weatherfish, and another attraction index $g r$ for yellowtail. The indexes $a$ and $g r$ were estimated by a method described in previous papers. Most of the vegetables tested ( 39 breeds from 36 kinds of vegetables) showed, to some extent, attraction activities for these three animals tested. The highest activity was observed in soybean Glycine max for black abalone, cabbage Brassica oleracea var. capitata (Nakawase 2) for weatherfish, and onion Allium cepa for yellowtail. The attraction activities of these vegetables clearly depended on the concentrations used.
\end{abstract}

Key words: attraction, vegetables, aquatic animals, behavior, abalone, oriental weatherfish, yellowtail

We examined the attraction activities of terrestrial food substances such as spices, herbal drugs, and fruits, which are foreign to aquatic environments, toward black abalone Haliotis discus, oriental weatherfish Misgurnus anguillicaudatus, and yellowtail Seriola quinqueradiata by behavioral experiments..$^{1-6)}$ The effectiveness of several spices, herbal drugs, and fruits has been proved to induce exploratory and feeding behaviors by tank experiments for the three aquatic animals described above. ${ }^{1-6)}$

To find attractants from easily available vegetables, similar experiments using the same target species were conducted, and the results are described in the present paper.

\section{Materials and Methods}

\section{Test Animals}

Young black abalone of $1.5 \mathrm{~cm}$ average shell length were supplied by the Yamaguchi Prefecture Gaikai Fisheries Experimental Station. Fifty individuals were used for each test. Adult oriental weatherfish $8.2 \mathrm{~cm}$ average body length were obtained from a private aquaculture farm in Shiga Prefecture and 60 individuals were used in each test. Juvenile yellowtails $4.9 \mathrm{~cm}$ average fork length were obtained from an aquaculture farm of Senzaki Cooperative in Yamaguchi Prefecture. One hundred and fifty three fish were accommodated in a test tank and the experiments were performed with the same fish. During the experimental period, 113 individuals were successively removed as they grew too big for testing in the test tank. Maintenance of the test animals and the procedures for the experiments were the same as described in previous papers. ${ }^{7-9)}$

\section{Test Samples}

Thirty-nine breeds from 36 kinds of vegetables tested are listed in Table 1. Each test sample was prepared as follows. Edible portions of the vegetable were thoroughly chopped into small pieces and homogenized with a food processor and an Ultra-Turrax homogenizer (Janke \& Kunkel GmbH \& Co., KG, Germany) in an equal volume of deionized water. The homogenate was then centrifuged for $15 \mathrm{~min}$ at $8,000 \times g$ below $5^{\circ} \mathrm{C}$ and the supernatant was filtered through a filter paper (Advantec Toyo No. 2). The filtrate was, if necessary, adjusted to $\mathrm{pH} 6.5$ by a dilute sodium hydroxide or hydrochloric acid solution and stored at $-40^{\circ} \mathrm{C}$ before use.

\section{Estimation of Attraction Indexes}

Attraction indexes, the A.I. $a$ for both abalone and oriental weatherfish, and the A.I. $g r$ for yellowtail were estimated by the methods described in the previous papers. ${ }^{7-9)}$ Briefly, the estimation of A.I. $a$ and A.I. $g r$ was based on applying the remained and the entered or left time-courses obtained from a behavioral experiment to a logistic curve $y=g /\{1+\exp [-r(x-a)]\}$. In respective tests, three test samples were chosen arbitrarily from the 39 , and set in the test compartment of three channels arranged arbitrarily out of the four in the test tank (one channel being left as a dummy). Statistical significance was evaluated using the chi-square test. A.I. $a$ and A.I. gr correspond to the average remained time $(\mathrm{min})$ and the number of locomotive fish at the coefficient $a$, respectively. A crumpled gauze (a sheet of $25 \mathrm{~cm} \times 25 \mathrm{~cm}$ ) in which $7.5 \mathrm{ml}$ of test sample was absorbed was set in each of the four compartments in the test tank to observe the behavior of the animals. Attraction activities of three arbitrary test samples and a dummy were measured at a time. 
Table 1. Terrestrial vegetables tested

\begin{tabular}{|c|c|c|}
\hline Common name & Japanese name & Botanical name \\
\hline Aralia family & Ukogika & Araliaceae \\
\hline Udo & Udo & Aralia cordata \\
\hline Arum family & Satoimoka & Araceae \\
\hline Taro & Satoimo & Colocasia esculenta \\
\hline Bean family & Marneka & Leguminosae \\
\hline Broad bean & Soramame & Vicia faba \\
\hline Pea & Akaendoumame & Pisum sativum \\
\hline Soybean & Edamame & Glycine $\max$ \\
\hline Ginger family & Shougaka & Zingiberaceae \\
\hline Ginger & Shouga & Zingiber officinale \\
\hline Mioga & Myouga & Zingiber mioga \\
\hline Gourd family & Urika & Cucurbitaceae \\
\hline Cucumber & Kyuurí & Cucumis sativus \\
\hline Grass family & Ineka & Gramineae \\
\hline Bamboo shoot & Takenoko & Phyllostachys bambusoides \\
\hline Lily family & Yurika & Liliaceae \\
\hline Chinese chive & Nira & Allium tuberosum \\
\hline Onion & Tamanegi & Allium cepa \\
\hline Rakkyo & Rakkyo & Allium chinense \\
\hline Mallow family & Aoika & Malvaceae \\
\hline Gumbo & Okura & Abelmoschus esculentus \\
\hline Mint family & Sisoka & Labiatae \\
\hline Perilla (I) & Siso & Perilla ocymoides \\
\hline Perilla (II) & Aosisomejiso & Perilla ocymoides \\
\hline Mustard family & Aburanaka & Cruciferae \\
\hline Broccoli & Burokkori & Brassica oleracea var. italica \\
\hline Cabbage (1) & Kyabetu & Brassica oleracea var. capitata (Nakawase 2) \\
\hline Cabbage (II) & Kyabetu & Brassica oleracea var. capitata (Kinsyun) \\
\hline Cauliflower & Karifurawa & Brassica oleracea var. botrytis \\
\hline Japanese radish & Daikon & Raphanus sativus var. longipinnatus \\
\hline Pakchoi & Chingensai & Brassica campestris var. chinensis \\
\hline Turnip & Kabu & Brassica campestris \\
\hline Wasabi & Wasabi & Eutrema wasabi \\
\hline Osmund family & Zenmaika & Osmundaceae \\
\hline Zen mai & Zenmai & Osmunda japonica \\
\hline Parsley family & Serika & Umbelliferae \\
\hline Carrot & Ninjin & Daucus carota \\
\hline Celery & Serori & Apium graveolens var. dulce \\
\hline Japanese hornwort & Mituba & Cryptotaenia japonica \\
\hline Parsley & Paseri & Petroselinum crispum \\
\hline Water dropwort & Seri & Oenanthe japonica \\
\hline Potato family & Nasuka & Solanaceae \\
\hline Eggplant & Nasu & Solanum melongena \\
\hline Irish potato $(\mathrm{I})^{*_{1}}$ & Jagaimo & Solanum tuberosum \\
\hline Irish potato $(11)^{*_{3}}$ & Jagaimo & Solanum tuberosum \\
\hline Sunflower family & Kikuka & Compositae \\
\hline Edible burdock (I) ${ }^{*}$ & Gobou & Arctium lappa \\
\hline Edible burdock (II) ${ }^{* 2}$ & Gobou & Arctium lappa \\
\hline Garland chrysanthemum & Shungiku & Chrysanthemum coronarium \\
\hline Head lettuce & Retasu & Lactuca sativa \\
\hline Japanese butterbur & Fuki & Petasites japonicus \\
\hline Leaf lettuce & Saniretasu & Lactuca sativa \\
\hline Mugwort & Yomogi & Artemisia princeps \\
\hline
\end{tabular}

*1 Product in Shimonoseki, Japan.

*2 Product in Takinokawa, Japan.

*3 Product in Atsugi, Japan.

\section{Determination of Free Amino Acids}

Free amino acid contents in the vegetable extracts were determined by high-performance liquid chromatography (HPLC). The HPLC was performed with a Hitachi L-6000 series by the OPA method described in the Hitachi HPLC Instruction Manual.

\section{Results and Discussion}

The calculated values of the attraction indexes of vegetable samples for the three test animals are listed in Table 2. The values in this table can be compared only among those in the same series No. Test samples with a higher value of attraction index than that of the dummy were regarded to 
Table 2. Attraction activities of vegetables

\begin{tabular}{|c|c|c|c|c|}
\hline \multirow{2}{*}{\multicolumn{2}{|c|}{ No. Vegetables }} & \multicolumn{3}{|c|}{ Attraction activities } \\
\hline & & $\begin{array}{l}\text { Abalone } \\
\text { (A.I. a) }\end{array}$ & $\begin{array}{l}\text { Oriental weatherfish } \\
\text { (A.I. a) }\end{array}$ & $\begin{array}{l}\text { Yellowtail } \\
\text { (A.I. gr) }\end{array}$ \\
\hline \multirow{4}{*}{1} & (Dummy & 5.1 & 2.8 & $167.8^{7}$ \\
\hline & Garland chrysanthemum & 5.0 & 6.4 & 158.1 \\
\hline & Japanese hornwort & 5.5 & 8.7 & 134.0 \\
\hline & Water dropwor: & 7.4 & 2.9 & 128.4 \\
\hline \multirow{4}{*}{2} & (Dummy & 5.7 & 1.9 & 181.4 \\
\hline & Chinese chive & 4.6 & 3.5 & 173.5 \\
\hline & Mugwort & 7.9 & 3.9 & 118.5 \\
\hline & Parsley & 6.0 & 12.2 & 166.1 \\
\hline \multirow{4}{*}{3} & Durnmy & 6.3 & 2.0 & 32.6 \\
\hline & Cabbage (I) & 11.8 & 3.6 & 53.7 \\
\hline & Leaf lettuce & 10.0 & 2.3 & 44.2 \\
\hline & Head lettuce & 4.0 & 2.9 & 31.1 \\
\hline \multirow{4}{*}{4} & Dummy & $4.6 ?$ & $2.5^{?}$ & 68.7 \\
\hline & Cabbage (II) & 6.0 & 3.1 & 122.2 \\
\hline & Perilla (I) & 5.3 & 2.3 & 142.5 \\
\hline & Perilla (II) & 5.2 & 2.2 & 79.9 \\
\hline \multirow{4}{*}{5} & Dummy & 5.7 & 1.7 & 73.8 \\
\hline & Japanese butterbur & 5.5 & 1.8 & 75.7 \\
\hline & Udo & 6.9 & 3.1 & 37.9 \\
\hline & Zen mai & 5.0 & 2.1 & 38.3 \\
\hline \multirow{4}{*}{6} & Dummy & 5.3 & 2.9 & 166.9 \\
\hline & Bamboo shoot & 6.3 & 3.2 & 88.2 \\
\hline & Onion & 6.4 & 3.7 & 191.6 \\
\hline & Rakkyo & 4.4 & 4.5 & 181.2 \\
\hline \multirow{4}{*}{7} & CDummy & 4.4 & 0.6 & 65.1 \\
\hline & Edible burdock (I) & 4.1 & 3.7 & 51.5 \\
\hline & Turnip & 6.2 & 2.8 & 121.0 \\
\hline & Wasabi & 9.6 & 1.3 & 12.5 \\
\hline \multirow{4}{*}{8} & Dummy & $5.4^{?}$ & 1.2 & 8.6 \\
\hline & Carrot & $5.2 \%$ & 2.1 & 17.3 \\
\hline & Irish potato (I) & 6.7 & 2.5 & 15.2 \\
\hline & Japanese radish & $4.0^{?}$ & 2.9 & 26.2 \\
\hline \multirow{4}{*}{9} & Dummy & 3.8 & 2.6 & 94.9 \\
\hline & Edible burdock (II) & 3.1 & 3.8 & 51.4 \\
\hline & Ginger & 5.8 & 3.3 & 29.1 \\
\hline & Irish potato (II) & 4.8 & 2.0 & 70.8 \\
\hline \multirow{4}{*}{10} & (Dummy & 6.2 & 1.8 & $71.3^{?}$ \\
\hline & $\int$ Broad bean & 8.0 & 2.9 & 79.8 \\
\hline & Pea & 9.1 & 2.1 & 65.7 \\
\hline & Soybean & 10.3 & 2.2 & 105.1 \\
\hline \multirow{4}{*}{11} & (Dummy & $9.8^{?}$ & 3.3 & 35.3 \\
\hline & Cucumber & 6.5 & 2.3 & 80.0 \\
\hline & Eggplant & 9.9 & 3.2 & 46.7 \\
\hline & Gumbo & 6.3 & 1.9 & 35.2 \\
\hline \multirow{4}{*}{12} & Dummy & 5.0 & 5.6 & 93.5 \\
\hline & Celery & $8.4^{7}$ & $3.5^{?}$ & 105.2 \\
\hline & Mioga & 4.5 & 6.6 & 106.7 \\
\hline & Taro & 4.9 & $3.6^{?}$ & 75.2 \\
\hline \multirow{4}{*}{13} & (Dummy & 10.9 & 3.2 & 168.1 \\
\hline & Broccoli & $9.6 ?$ & $4,0^{?}$ & 123.1 \\
\hline & Cauliflower & 8.0 & 4.2 & 138.0 \\
\hline & Pakchoi & 5.2 & 4.7 & 143.1 \\
\hline
\end{tabular}

A.l. $a$ and $g r$ are derived from the coefficients of a logistic curve $y=g /(1+\exp [-r(x-a)]\}$ being applied to remained, and entered or left time-courses for black abalone and oriental weatherfish, and yellowtail, respectively. The indexes, which were estimated from the series of data showing good fitness, $\operatorname{Pr}\left(\chi^{2}>\chi \chi^{2} . b_{0}\right)$ were listed, otherwise attached with s mark *h"t" 
be effective in attraction. For abalone, the following vegetables were effective in attraction: Japanese hornwort, water dropwort, mugwort, parsley, cabbage (I), leaf lettuce, cabbage (II), perilla (I), perilla (II), udo, bamboo shoot, onion, turnip, wasabi, Irish potato (I), ginger, Irish potato (II), broad bean, pea, soybean, and celery. Among them, leaf lettuce, cabbage (I), wasabi, soybean, and celery were most attractive.

For oriental weatherfish, the following vegetables were effective in attraction: garland chrysanthemum, Japanese hornwort, Chinese chive, mugwort, parsley, cabbage (I), leaf lettuce, head lettuce, cabbage (II), udo, zen mai, bamboo shoot, onion, rakkyo, edible burdock (I), turnip, wasabi, carrot, Irish potato (I), Japanese radish, edible burdock (II), ginger, broad bean, pea, soybean, mioga, broccoli, cauliflower, and pakchoi. Among them, garland chrysanthemum, Japanese hornwort, mugwort, parsley, cabbage (I), edible burdock (I), turnip, wasabi, Irish potato (I), and Japanese radish were most attractive.

For yellowtail, the following vegetables were effective in

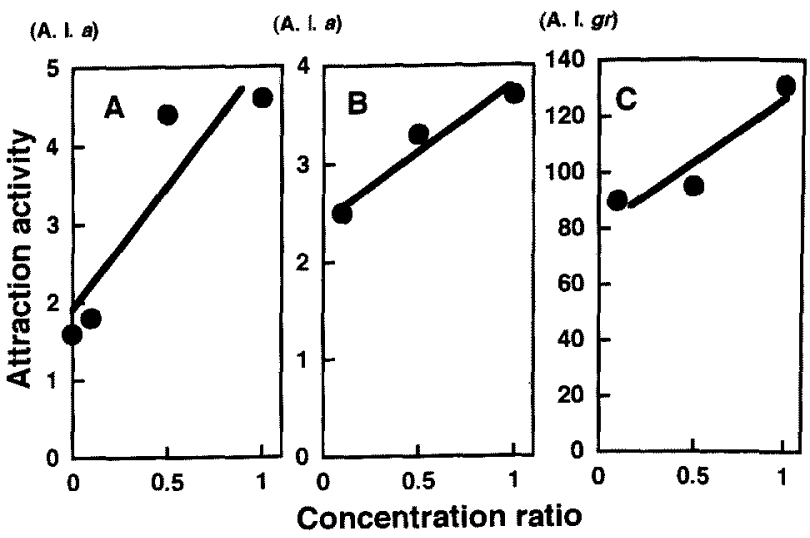

Fig. 1. Effects of concentrations on attraction activities, (A) soybean for black abalone, (B) cabbage (I) for oriental weatherfish, (C) onion for yellowtail.

Attraction activity was examined for three relative concentrations diluted from the original vegetable extract.

Table 3. Attraction activities of active vegetables for abalone

\begin{tabular}{|c|c|c|c|c|}
\hline \multicolumn{2}{|c|}{ No. Vegetables } & $\begin{array}{l}\text { Attraction activities* } \\
\text { (A.I. } a \text { ) }\end{array}$ & No. Vegetables & $\begin{array}{l}\text { Attraction activities } \\
\text { (A.I. } a \text { ) }\end{array}$ \\
\hline \multirow{4}{*}{14} & Dummy & $5.5 ?$ & (Dummy & - \\
\hline & Cabbage (I) & 16.0 & ${ }_{15}$ Irish potato (I) & 5.1 \\
\hline & Mugwort & 9.0 & 15 Onion & $3.7^{?}$ \\
\hline & Water dropwort & 6.4 & Wasabi & 6.3 \\
\hline \multirow{4}{*}{16} & (Dummy & $2.5^{?}$ & (Dummy & - \\
\hline & Celery & 5.8 & 17 Cabbage (I) & 5.3 \\
\hline & Ginger & $2.4 ?$ & 17 Soybean & 8.6 \\
\hline & Soybean & $7.4 ?$ & Wasabi & $7.4 ?$ \\
\hline
\end{tabular}

* legends in Table 2. Nos. 14-16: intermediate selection. No. 17: final selection.

Table 4. Attraction activities of active vegetables for oriental weatherfish

\begin{tabular}{|c|c|c|c|c|}
\hline No. & Vegetables & $\begin{array}{l}\text { Attraction activities* } \\
\text { (A.I. a) }\end{array}$ & No. Vegetables & $\begin{array}{l}\text { Attraction activities* } \\
\text { (A.I. a) }\end{array}$ \\
\hline \multirow{4}{*}{18} & (Dummy & 3.0 & \multirow{4}{*}{$19\left\{\begin{array}{l}\text { Edible burdock (I) } \\
\text { Rakkyo } \\
\text { Udo }\end{array}\right.$} & 1.9 \\
\hline & Cabbage (I) & 7.7 & & 3.7 \\
\hline & Japanese hornwort & 3.9 & & $3.0^{?}$ \\
\hline & Parsley & 5.9 & & $2.6^{?}$ \\
\hline \multirow{4}{*}{20} & (Dummy & 2.7 & \multirow{4}{*}{$21\left\{\begin{array}{l}\text { Cabbage (I) } \\
\text { Edible burdock (1) } \\
\text { Japanese radish }\end{array}\right.$} & 1.0 \\
\hline & Japanese radish & 4.3 & & 4.5 \\
\hline & Mioga & 2.9 & & 3.1 \\
\hline & (Pakchoi & 3.9 & & 4.3 \\
\hline
\end{tabular}

* legends in Table 2. Nos. 18-20: intermediate selection. No. 21: final selection.

Table 5. Attraction activities of active vegetables for yellowtail

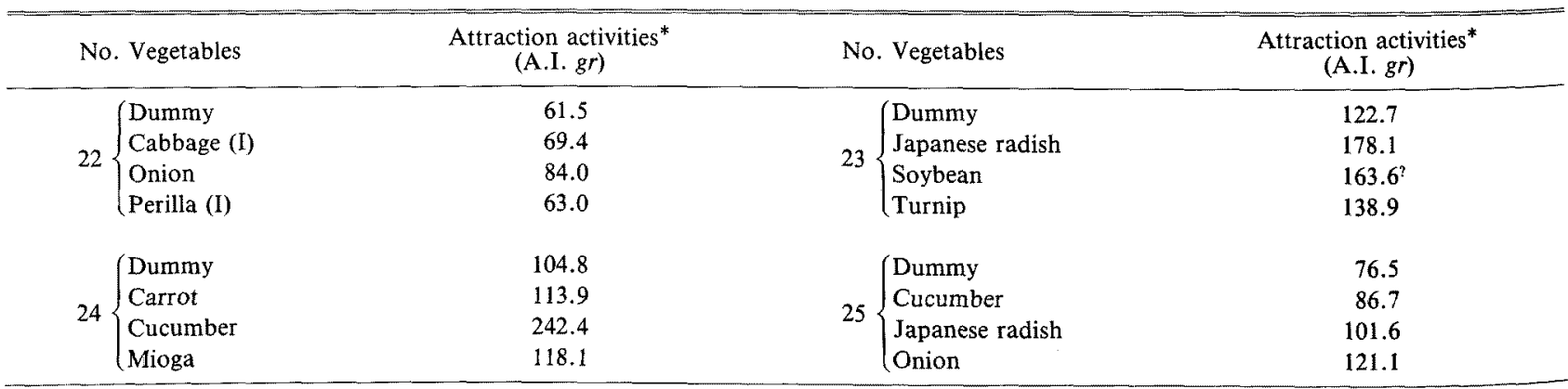

* legends in Table 2. Nos. 22-24: intermediate selection. No. 25: final selection. 
attraction: cabbage (I), leaf lettuce, cabbage (II), perilla (I), duce any unusual behavior pattern during the experiment, perilla (II), onion, rakkyo, turnip, carrot, Irish potato (I), and the test individuals swam into and away from the test Japanese radish, broad bean, soybean, cucumber, egg- compartment as in the test of attractants previously plant, celery, and mioga. Among them, perilla (I), onion, reported..$^{1-6)}$

carrot, Japanese radish, and cucumber were most attractive.

Furthermore, to determine the most effective vegetables

The vegetables that were effective in attraction did not infor each test animal, 9 vegetables, which showed strong effects in Table 2, were sorted from the 39, and tests were

Table 6. Attraction magnitude and organoleptic taste and/or flavor of vegetables tested

\begin{tabular}{|c|c|c|c|c|}
\hline \multirow{2}{*}{ Common name } & \multicolumn{3}{|c|}{ Attraction magnitude ${ }^{*_{1}}$} & \multirow{2}{*}{ Taste or taste with flavor } \\
\hline & $\mathrm{A}^{* 2}$ & $\mathrm{O}^{* 2}$ & $Y^{* 2}$ & \\
\hline \multicolumn{5}{|l|}{ Aralia family } \\
\hline Udo & + & + & & Astringent \\
\hline \multicolumn{5}{|l|}{ Arum family } \\
\hline \multirow{2}{*}{\multicolumn{5}{|c|}{ Bean family }} \\
\hline & & & & \\
\hline Broad bean & + & + & + & Very slightly sweet \\
\hline Pea & + & + & & Slightly sweet \\
\hline Soybean & ++ & + & + & Slightly sweet \\
\hline \multicolumn{5}{|l|}{ Ginger family } \\
\hline Ginger & + & + & & Bitter \\
\hline Mioga & & + & + & Astringent \\
\hline \multicolumn{5}{|l|}{ Gourd family } \\
\hline Cucumber & & & $+t$ & Slightly sweet \\
\hline \multicolumn{5}{|l|}{ Grass family } \\
\hline Bamboo shoot & + & + & & Slightly sweet \\
\hline \multicolumn{5}{|l|}{ Lily family } \\
\hline Chinese chive & & + & & Strongly bitter \\
\hline Onion & + & + & $t+$ & Strongly sweet \\
\hline Rakkyo & & + & + & Strongly sweet \\
\hline \multicolumn{5}{|l|}{ Mallow family } \\
\hline Gumbo & & & & No taste, strong grassy flavor \\
\hline \multicolumn{5}{|l|}{ Mint family } \\
\hline Perilla (I) & + & & ++ & Astringent \\
\hline Perilla (II) & + & & + & Astringent \\
\hline \multicolumn{5}{|l|}{ Mustard family } \\
\hline Broccoli & & + & & Bitter, sweet flavor \\
\hline Cabbage (I) & $+t$ & ++ & + & Slightly sweet \\
\hline Cabbage (II) & + & + & + & Slightly sweet \\
\hline Cauliffower & & + & & Bitter, sweet flavor \\
\hline Japanese radish & & ++ & ++ & Slightly sweet \\
\hline Pakchoi & & + & & Bitter, grassy flavor \\
\hline Turnip & + & $+t$ & + & Slightly sweet \\
\hline Wasabi & ++ & ++ & & Hot, pungent \\
\hline \multicolumn{5}{|l|}{ Osmund family } \\
\hline Zen mai & & + & & Bitter \\
\hline \multicolumn{5}{|l|}{ Parsley family } \\
\hline Carrot & & + & $t+$ & Slightly sweet \\
\hline Celery & ++ & & + & Astringent \\
\hline Japanese hornwort & + & ++ & & Bitter \\
\hline Parsley & + & ++ & & Bitter \\
\hline Water dropwort & + & & & Bitter \\
\hline \multicolumn{5}{|l|}{$\begin{array}{l}\text { Potato family } \\
\text { Port }\end{array}$} \\
\hline Eggplant & & & + & Slightly sweet \\
\hline Irish potato (I) & + & ++ & + & Slightly sweet \\
\hline Irish potato (II) & + & & & Slightly sweet \\
\hline \multicolumn{5}{|l|}{ Sunflower family } \\
\hline Edible burdock (I) & & $+t$ & & Slightly sweet \\
\hline Edible burdock (II) & & + & & Slightly sweet \\
\hline Garland chrysanthemum & & ++ & & Strongly bitter \\
\hline Head lettuce & & + & & Slightly sweet \\
\hline Japanese butterbur & & & & Bitter, sweet flavor \\
\hline Leaf lettuce & ++ & + & + & Slightly sweet, grassy flavor \\
\hline Mugwort & + & $+t$ & & Astringent \\
\hline
\end{tabular}

* +; weak, + +; strong.

" A; black abalone, $\mathrm{O}$; oriental weatherfish, $\mathrm{Y}$; yellowtail. 
repeated using the same method as the previous one, i.e., for combinations of 3 out of the 9 (Tables 3, 4, and 5). As the results of the final combination test (Nos. 17, 21, and 25 in Tables 3, 4, and 5, respectively), it was found that soybean, cabbage (I), and onion were the most active for abalone, oriental weatherfish, and yellowtail, respectively. The effect of concentration of the three vegetable extracts on attraction activity was tested for corresponding animals (Fig. 1). Attraction activities of the three concentrations of each vegetable extract with the dummy were measured. At- traction activities for all the three extracts increased with increasing concentration over the concentration range investigated. The results of the experiments are summarized together with the organoleptic taste of the vegetable extracts in Table 6, which indicate that attractant vegetables for all the test animals were broad bean, soybean, onion, cabbage (I), cabbage (II), turnip, Irish potato (I), and leaf lettuce.

There have been several studies on the attractive effect of constituents in food "plants" that are foreign to aquat-

Table 7. Contents of free amino acids in vegetable extracts tested $(\mathrm{mg} / 100 \mathrm{~m} /$ test solution)

\begin{tabular}{|c|c|c|c|c|c|c|c|c|c|c|c|c|c|c|c|c|c|c|}
\hline Common name & Asp & Thr & Ser & Glu & Pro & Gly & $\mathrm{Ala}$ & Cys & Val & Met & Ile & Leu & Tyr & Phe & Lys & His & Arg & Total \\
\hline \multicolumn{19}{|l|}{ Aralia family } \\
\hline Udo & 15.4 & 112.4 & 1.6 & 83.4 & - & 0.6 & 2.6 & - & 2.8 & - & 3.4 & 5.0 & - & - & - & 6.6 & - & 233.8 \\
\hline \multicolumn{19}{|l|}{ Arum family } \\
\hline Taro & 15.2 & 11.2 & 11.8 & 18.0 & - & 1.4 & 7.4 & - & 6.0 & 7.2 & 3.6 & 16.2 & - & 7.8 & 3.2 & 2.2 & - & 111.2 \\
\hline \multicolumn{19}{|l|}{ Bean family } \\
\hline Broad bean & 37.0 & 52.4 & 18.0 & 49.0 & - & 9.4 & 70.4 & - & 19.6 & - & 48.6 & 15.4 & 26.6 & 26.2 & 33.8 & 28.0 & 229.6 & 664.0 \\
\hline Pea & 10.6 & 65.4 & 34.4 & 168.2 & - & 31.4 & 46.8 & - & 38.8 & 7.2 & 65.2 & 33.4 & 28.0 & 31.8 & 50.4 & 28.8 & 272.2 & 912.6 \\
\hline Soybean & 51.6 & 36.2 & 39.4 & 10.8 & 25.6 & 24.4 & 81.4 & - & 21.6 & - & 27.4 & 22.6 & -1 & 104.6 & 27.8 & 50.2 & 93.0 & 616.6 \\
\hline \multicolumn{19}{|l|}{ Ginger family } \\
\hline Ginger & 21.0 & 23.0 & 16.8 & 11.0 & - & 2.0 & 9.6 & - & 5.6 & 13.2 & 38.4 & 39.6 & - & 5.0 & 6.8 & 2.0 & 1.8 & 195.8 \\
\hline Mioga & 11.8 & 11.2 & 9.2 & 7.6 & - & 4.0 & 2.0 & - & 1.4 & - & 1.0 & 1.2 & - & 2.4 & 0.8 & 1.4 & - & 54.0 \\
\hline \multicolumn{19}{|l|}{ Gourd family } \\
\hline Cucumber & 3.0 & 29.6 & 8.0 & 13.6 & 11.4 & 6.4 & 3.8 & - & 3.6 & - & 5.2 & 5.6 & - & 4.4 & 3.6 & 2.0 & - & 100.2 \\
\hline \multicolumn{19}{|l|}{ Grass family } \\
\hline Bamboo shoot & 17.6 & 28.4 & 27.0 & 29.4 & - & 3.8 & 11.0 & - & 17.2 & - & 15.0 & 20.8 & 81.4 & 17.6 & 21.8 & 15.6 & 15.6 & 322.2 \\
\hline \multicolumn{19}{|c|}{ 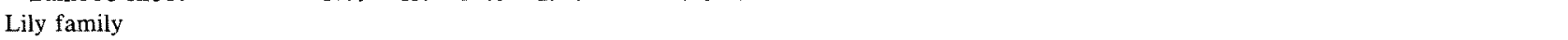 } \\
\hline Chinese chive & 19.0 & 27.6 & 4.8 & 78.8 & - & 5.8 & 24.8 & - & 11.0 & - & 16.0 & 14.2 & - & 10.2 & 28.4 & 4.4 & 9.0 & 254.0 \\
\hline Onion & 9.4 & 26.2 & 7.0 & 33.6 & - & 1.0 & 4.8 & - & 7.0 & - & 7.8 & 15.4 & 15.2 & 9.2 & 23.6 & 10.6 & 41.2 & 212.0 \\
\hline Rakkyo & 12.0 & 37.2 & 13.6 & 118.6 & 13.4 & 2.4 & 17.4 & - & 2.6 & 11.0 & 4.0 & 3.4 & - & 14.4 & 30.6 & 14.2 & 141.2 & 436.0 \\
\hline \multicolumn{19}{|l|}{ Mallow family } \\
\hline Gumbo & 48.6 & 69.8 & 12.8 & 19.4 & 2.4 & 2.8 & 17.6 & - & 5.4 & - & 5.4 & 2.6 & - & 8.6 & 2.4 & 5.2 & - & 203.0 \\
\hline \multicolumn{19}{|l|}{ Mint family } \\
\hline Perilla (I) & 5.8 & 3.2 & 2.6 & 20.6 & - & 2.8 & 6.4 & - & - & - & 2.6 & 1.8 & - & 5.2 & - & - & - & 51.0 \\
\hline Perilla (II) & 9.6 & 2.4 & 1.8 & 12.2 & - & 1.2 & 6.6 & - & - & - & 1.0 & 1.2 & - & 3.2 & - & - & - & 39.2 \\
\hline \multicolumn{19}{|l|}{ Mustard family } \\
\hline Broccoli & 73.6 & 187.8 & 91.8 & 110.0 & 15.6 & 7.8 & 45.4 & - & 76.6 & - & 34.2 & 22.0 & 13.0 & 83.6 & 44.8 & 47.0 & 153.4 & 1006.6 \\
\hline Cabbage (I) & 20.2 & 93.8 & 17.4 & 13.0 & - & 3.8 & 25.0 & - & 11.8 & - & 16.8 & 10.4 & - & 6.0 & 19.0 & 12.2 & 21.0 & 270.4 \\
\hline Cabbage (II) & 19.8 & 45.8 & 12.6 & 6.8 & - & 2.2 & 15.8 & - & - & - & 13.8 & 8.0 & - & 4.6 & 4.6 & 9.0 & 16.0 & 159.0 \\
\hline Cauliflower & 35.8 & 27.2 & 15.4 & 40.6 & 9.2 & 5.2 & 30.0 & - & 15.8 & - & 13.2 & 16.2 & 7.0 & 8.8 & 15.8 & 3.6 & - & 243.8 \\
\hline Japanese radish & 15.0 & 48.8 & 6.8 & 4.6 & - & 2.4 & 7.2 & - & 5.8 & - & 5.0 & 2.2 & - & 2.2 & 3.4 & 3.0 & 12.6 & 119.0 \\
\hline Pakchoi & 19.2 & 14.6 & 7.0 & 7.4 & - & 1.8 & 9.0 & - & 9.0 & - & 4.8 & 6.8 & - & 6.8 & - & 3.0 & - & 89.4 \\
\hline Turnip & 12.6 & 14.8 & 7.0 & 14.6 & - & 4,2 & 6.8 & - & 4.2 & 40.4 & 11.6 & 5.0 & - & 14.4 & 3.6 & 2.4 & 7.4 & 149.0 \\
\hline Wasabi & 20.0 & 45.0 & 12.4 & 31.4 & - & 1.4 & 13.2 & - & 6.6 & - & 6.6 & 3.0 & - & 4.8 & 5.8 & 3.0 & 6.0 & 159.2 \\
\hline \multicolumn{19}{|l|}{ Osmund family } \\
\hline Zen mai & 5.2 & 27.6 & 1.8 & 46.8 & - & 0.4 & 6.2 & - & - & - & 188.6 & - & 14.8 & 46.8 & - & - & - & 338.2 \\
\hline \multicolumn{19}{|l|}{ Parsley family } \\
\hline Carrot & 25.0 & 54.6 & 6.8 & 1.2 & - & 1.6 & 21.0 & - & 6.0 & 13.6 & 5.2 & 3.0 & - & 4.8 & 4.0 & - & 4.8 & 151.6 \\
\hline Celery & 7.8 & 57.4 & 6.6 & 1.8 & - & 1.0 & 5.6 & - & 13.6 & - & 10.6 & 8.0 & - & 9.2 & 5.4 & 5.2 & - & 132.2 \\
\hline Japanese hornwort & 4.0 & 1.6 & 2.6 & 2.0 & - & 1.0 & 4.4 & - & - & - & 2.4 & 2.2 & - & 7.0 & - & - & - & 27.2 \\
\hline Parsley & 19.4 & 10.2 & 4.6 & 25.4 & - & 3.4 & 15.4 & - & 14.0 & - & 12.6 & 24.0 & - & 15.8 & 16.8 & 2.4 & 10.0 & 174.0 \\
\hline Water dropwort & 10.4 & 17.4 & 0.4 & 7.6 & - & 0.8 & 5.6 & - & 3.8 & - & 47.6 & 11.0 & - & 58.8 & - & - & 88.2 & 251.6 \\
\hline \multicolumn{19}{|l|}{ Potato family } \\
\hline Eggplant & 15.8 & 26.2 & 3.6 & 3.2 & - & 1.6 & 3.2 & - & 4.8 & 14.8 & 4.6 & 2.8 & 109.4 & 9.8 & 3.8 & 3.6 & - & 207.2 \\
\hline Irish potato (I) & 15.6 & 73.6 & 7.4 & 7.0 & - & 2.8 & 8.2 & - & 25.2 & 7.8 & 9.6 & 6.2 & - & 23.2 & 9.8 & 5.0 & 40.0 & 241.4 \\
\hline Irish potato (II) & 37.4 & 94.4 & 13.2 & 24.4 & - & 4.6 & 13.2 & - & 40.6 & 13.2 & 20.0 & 11.4 & - & 43.6 & 23.0 & 10.6 & 61.4 & 411.0 \\
\hline Sunflower family & & & & & & & & & & & & & & & & & & \\
\hline Edible burdock (I) & 6.8 & 1.8 & 1.6 & 5.4 & - & 0.6 & 1.8 & - & - & - & 2.6 & 1.2 & - & 8.2 & - & - & 6.2 & 36.2 \\
\hline Edible burdock (II) & 35.2 & 17.8 & 1.4 & 165.6 & - & 2,0 & 8.6 & - & 5.8 & - & 9.0 & 4.8 & - & 16.6 & 2.2 & 5.8 & 130.2 & 405.0 \\
\hline Garland chrysanthemum & 4.1 & 1.6 & 1.2 & 3.4 & - & 0.6 & 1.2 & - & - & 9.2 & 2.8 & 1.2 & - & - & - & - & - & 25.3 \\
\hline Head lettuce & 5.0 & 23.4 & 5.0 & 4.8 & - & 1.2 & 2.6 & - & 2.4 & - & 3.8 & 2.6 & 2.6 & 5.8 & 1.6 & 0.8 & - & 61.6 \\
\hline Japanese butterbur & 1.8 & 2.2 & 1.8 & 4.8 & - & 0,4 & 11.4 & - & 1.0 & - & 1.2 & 0.6 & - & 2.6 & - & - & - & 27.8 \\
\hline Leaf lettuce & 6.2 & 3.6 & 2.2 & 9.4 & - & 0.6 & 3.2 & - & - & - & 3.2 & 2.4 & - & - & - & - & - & 30.8 \\
\hline Mugwort & 5.8 & 3.2 & 2.2 & 10.6 & - & 0.6 & 5.0 & - & 2.0 & - & 2.6 & 4.0 & - & 8.6 & - & 0.8 & - & 45.4 \\
\hline
\end{tabular}


ic environments..$^{1-3,10-14)}$ In the previous papers, we have examined attraction activities of various spices, herbal drugs and fruits for black abalone, oriental weatherfish, and yellowtail..$^{1-6)}$ Most of the samples were found to be effective to some extent for the test animals. In the present study, all the vegetables tested except taro, Japanese butterbur, and gumbo also showed to some extent attraction activities for more than one test animal. All the Cruciferae vegetables tested ( 8 kinds) proved to be effective in attraction for oriental weatherfish and attraction activity was strong in half of these vegetables. This may suggest that the Cruciferae vegetables widely contain some specific attractive compound(s) for oriental weatherfish. However, no such strong effect was found for abalone nor yellowtail with vegetables of the family Cruciferae.

A kind of spice, anise Pimpinella anisum, attracted and stimulated rainbow trout Salmo gairdneri. ${ }^{11)}$ Furthermore, a kind of vegetable, lettuce Lactuca sativa var. longifolia, stimulated herbivorous fish Tilapia zillii, ${ }^{12,13)}$ snail Australorbis glabratus ${ }^{10)}$ and pond snail Lymnaea stagnalis. ${ }^{14)}$ From these studies, it is possible that organisms which are not distributed in the inhabitant environment of aquatic animals might contain effective constituents as feeding attractants or stimulants for the animals.

The majority of attractive fruit extracts for abalone, oriental weatherfish, and yellowtail were, to some extent, sweet and sour tastes as organoleptic to human in our previous study. ${ }^{4-6)}$ Sugars and their related compounds, which are sweet for human, were also attractive for aquatic animals. ${ }^{15-17)}$ These findings suggested that sweet compounds contained in the extracts attract the aquatic animals. In the present study, most of the vegetables that showed high attraction activity were, to some extent, sweet tasting for human (Table 6). On the other hand, the following vegetables having a specific taste and flavor for human also attracted the test animals: wasabi and celery for black abalone, wasabi, Japanese hornwort, parsley, garland chrysanthemum, and mugwort for oriental weatherfish, and perilla (I) for yellowtail. These vegetable extracts provide no sweet taste for human (Table 6). It is probable that the components which caused the specific tastes and flavors participate in the attraction activity.

The test animals abalone, oriental weatherfish, and yellowtail were attracted by such compounds contained in their feed as sugars, lipids, nitrogenous bases, nucleic acid-related compounds, proteins, amino acids, and/or others. ${ }^{18-22)}$ Amino acids that had high attraction activity were as follows: lysine, ornithine, and hydroxyproline for abalone; histidine, lysine, arginine, glycine, and alanine for oriental weatherfish; and histidine, glycine, threonine, and arginine for yellowtail. ${ }^{18-20)}$ The free amino acid contents in the vegetable extracts tested were determined to examine the relationship between the attraction activity and the amino acid contents (Table 7). For abalone, effective vegetable extracts contained lysine (in soybean, cabbage (I), wasabi, celery, and leaf lettuce) at relatively low concentrations, although only lysine was determined among the attractants of amino acids described above. On the contrary, for oriental weatherfish and yellowtail, effective vegetable extracts contained alanine (in cabbage (I) and Japanese hornwort), lysine (in cabbage (I)), or arginine (in edible burdock (I), cabbage (I), Japanese radish, and Irish potato
(I)), and threonine (in Japanese radish, cucumber, and carrot) or arginine (in onion) at relatively high concentrations among the attractants of amino acids described above, respectively. These findings suggested that these amino acids were responsible for attraction activities of vegetable extracts for oriental weatherfish and yellowtail, but not for abalone. On the other hand, it is reported that the herbivorous fish Tilapia zillii was strongly attracted by the following amino acids fractionated from lettuce: glutamic acid, aspartic acid, lysine, alanine, and serine. ${ }^{12)}$ These attractive amino acids therefore probably participate at least in the attraction activity of the vegetable for herbivorous fish.

Among the terrestrial vegetables listed in Table 1,2 specimens each of edible burdock and Irish potato were used from different places of production, while 2 specimens each of cabbage and perilla were also used from different breeds. The different attraction activities in these vegetables may be related to the different places of production or breed (Tables 2 and 6). Compositions in the vegetable extracts may delicately depend on the place of production, breed, maturity, or freshness. For examples, differences in the free amino acid compositions were observed in the present paper between the vegetables from different places of production or from different breeds. This difference in amino acid compositions and/or other components may affect the attraction activity.

In conclusion, most of the vegetables tested, which are foreign to aquatic animals, probably contain some attractants for black abalone, oriental weatherfish, and yellowtail.

\section{References}

1) K. Harada: Attraction activities of spices for oriental weatherfish and yellowtail. Nippon Suisan Gakkaishi, 56, 2029-2033 (1990).

2) K. Harada: Attraction activities of herbal crude drugs for abalone, oriental weatherfish, and yellowtail. Nippon Suisan Gakkaishi, 57, 2083-2088 (1991).

3) K. Harada: Part IV. Feeding and growth, in Abalone of the World (ed. by S. A. Shepherd, M. J. Tegner, and S. A. Guzmán del Próo), Fishing News Books, Oxford, 1992, pp. 193-200.

4) K. Harada and T. Miyasaki: Attraction activities of fruit extracts for the oriental weatherfish Misgurnus anguillicaudatus. Nippon Suisan Gakkaishi, 59, 1757-1762 (1993).

5) K. Harada and T. Miyasaki: Attraction activities of fruit flesh water extracts for yellowtail Seriola quinqueradiata. J. Shimonoseki Univ. Fish., 42, 73-80 (1994).

6) K. Harada and T. Miyasaki: Attraction activities of fruit flesh water extracts toward black abalone, Haliotis discus. Suisanzoshoku, $\mathbf{4 2}$, 171-177 (1994).

7) K. Harada and $O$. Kawasaki: The attractive effect of seaweeds based on the behavioral responses of young herbivorous abalone Haliotis discus. Nippon Suisan Gakkaishi, 48, 617-621 (1982).

8) K. Harada, M. Maéda, and K. Kobayashi: Application of logistic curve as index of the attractive effect of food for oriental weatherfish. Nippon Suisan Gakkaishi, 48, 21-29 (1982).

9) K. Harada: The attractive effect of food based on the behavioral responses of juvenile yellowtail Seriola quinqueradiata. Nippon Suisan Gakkaishi, 48, 1047-1054 (1982).

10) E. H. Michelson: Chemoreception with the snail Australorbis glabratus. Am. J. Trop. Med. Hyg., 9, 480-487 (1960).

11) M. Takei: Studies on fishes favorite foods-I. Feeding tests of carp, yellowtail, and rainbow trout. Bull. Tokai Reg. Fish. Res. Lab., No. 49, 119-129 (1967).

12) P. B. Johnsen and M. A. Adams: Chemical feeding stimulants for 
the herbivorous fish, Tilapia zillii. Comp. Biochem. Physial., 83A, 109-112 (1986)

13) M. A. Adams, P. B. Johnsen, and Zhou Hong-Qi: Chemical enhancement of feeding for the herbivorous fish Tilapia zillii. Aquaculture, 72, 95-107 (1988).

14) J. T. Goldschmeding and J. C. Jager: Feeding responses to sucrose in the pond snail Lymnaea stagnalis after nerve section and tentacle amputation. Nether. J. Zool., 23, 118-124 (1973).

15) K. Harada, T. Miyasaki, and Y. Tamura: Chemoattractant effects of sugars and their related compounds on black abalone Haliotis discus. Comp. Biochem. Physiol., 109A, 111-115 (1994).

16) K. Harada, T. Miyasaki, and Y. Tamura: Attractivity of sugars and related compounds for the oriental weatherfish Misgurnus anguillicaudatus. Fisheries Sci., 60, 643-645 (1994).

17) K. Harada, T. Miyasaki, and Y. Tamura: Attraction of yellowtail, Serioia quinqueradiata for sugars and related compounds. Suisan- zoshoku, 43, 51-55 (1995).

18) K. Harada and Y. Akishima: Feeding attraction activities of proteins, amino acids, lipids, and nitrogenous bases for abalone. Nippon Suisan Gakkaishi, 51, 2051-2058 (1985).

19) K. Harada: Feeding attraction activities of amino acids and nitrogeneous bases for oriental weatherfish. Nippon Suisan Gakkaishi, 51, 461-466 (1985).

20) K. Harada: Feeding attraction activities of amino acids and lipids for juvenile yellowtail. Nippon Suisan Gakkaishi, 51, 453-459 (1985).

21) K. Harada: Feeding attraction activities of nucleic acid-related compounds for abalone, oriental weatherfish and yellowtail. Nippon Suisan Gakkaishi, 52, 1961-1968 (1986).

22) K. Harada: Feeding attraction activities of L-dipeptides for abalone, oriental weatherfish and yellowtail. Nippon Suisan Gakkaishi, 55, 1629-1634 (1989). 